An analysis of the line-emission carried out by Mustel and myself has shown that a strong self-absorption takes place in flares. We were able to show in $\mathrm{I} 95^{2}$ that the $\mathrm{H} \alpha$ line is broadened by natural damping, whereas the other Balmer lines probably are broadened by the Stark effect. This can be seen from a comparison between theoretical and observed profiles. The data permit us to evaluate the flux of $L \alpha$ radiation and its selective pressure which appears to be sufficient for the ejection of hydrogen atoms with velocities up to $4000 \mathrm{~km}$. $/ \mathrm{sec}$.

A strong self-absorption takes place in the $\mathrm{H}$ and $\mathrm{K}$ lines too. The best agreement between theoretical and observed contours was obtained when assuming that the wings are broadened by natural damping and the central core by the Doppler effect (corresponding to $\mathrm{N}_{1}=5 . \mathrm{IO}^{15}, T_{\mathrm{kin}}=5.500$ and $v_{t}=0$ ). The minute to minute cinematography of flares and active regions shows the flare phenomenon to be an extremely dissipative process. For all the flares examined the increase in $\mathrm{H} \alpha$ line intensity is accompanied by a growth of the area. More than $30 \%$ of the flares show a marked and very rapid motion of luminous matter.

The larger the velocity of expansion of a flare, the higher is the maximum $\mathrm{H} \alpha$ intensity. The lifetime of a flare (halfwidth of light curve) increases as the square of its size. This is quite natural for any dissipative process. The surprisingly low optical depth $(0 \cdot 8)$ in the centre of $\mathrm{H} \alpha$ for the limb flare of I6 August I952 showed that recombinations are not responsible for the observed emission.

The resolution of flares and faculae into small grains of emission, the peculiar character of continuous as well as line-emission and the characteristic features of their development lead to the conclusion that some intense explosive process, may be nuclear process, might be responsible for these phenomena. The small size of the grains indicates high density of energy and reveals the possibility of high-density electric currents, but does not exclude the nuclear processes too. The observed sharp edge of emission from one side and the diffuse edge from the other side favours the conception of a shock wave.

The abundance of such elements as $\mathrm{Li}, \mathrm{Be}$ and Deuterium in the solar atmosphere also favours the conception of nuclear explosions.

\title{
5. CENTRE-LIMB EFFECTS IN SOLAR FLARES
}

\section{By L. GOLDBERG}

It is well established that ionospheric disturbances in the Earth's atmosphere are caused by ultra-violet radiation associated with solar flares. Until recently, the correlation between the two phenomena was less than precise, since a certain number of bright flares were not accompanied by sudden ionospheric disturbances. Dodson (I955) has pointed out, however, that not all ionospheric disturbances are sudden, and has introduced the new designation of gradual ionospheric disturbance (G.I.D.) to denote the slowly increasing type of disturbance that frequently follows the onset of a flare. She has discovered further that when the product of maximum $\mathrm{H} \alpha$ intensity and total flare area exceeds a certain threshold value, an ionospheric disturbance, either sudden or gradual, will almost certainly take place.

The probability is also high that the $L \alpha$ line is the ultra-violet radiation responsible for I.D.'s, since no other radiation of sufficient ionizing power appears capable of penetrating the atmosphere to the altitude of the D layer (Byram et al. I953; Goldberg, I954). If the $L \alpha$ line is indeed responsible for D-layer disturbances, it becomes of great importance to know its intensity during solar flares. It is for this reason that strong attempts will be made to observe the line from high-altitude rockets during solar flares at the time of the International Geophysical Year. While it is likely that at least some of the attempts will prove successful, it would obviously be extremely fruitful to develop a successful theory for the flare-emitting layer which would permit the calculation of the $\mathrm{L} \alpha$ intensity from observations of the Balmer series lines. 
Since no two flares behave exactly alike and since systematic and continuing observations of flare spectra have in the main been limited to the vicinity of $\mathrm{H} \alpha$, it seems hardly possible at the present time to derive unambiguous models for flare-emitting layers with anything like the precision that can be obtained for the photosphere (or even the chromosphere!). The most extensive compilations of photometric data on flares are those of Miss Dodson (1955), which consist of measurements of the central intensity of $\mathrm{H} \alpha$ for several hundred flares, and of Ellison (r950), which are based both upon measurements of $\mathrm{H} \alpha$ line-profiles and upon visual observations of $\mathrm{H} \alpha$ line-widths. Examination of these data reveals an almost bewildering diversity of phenomena which sometimes seem to defy unification.

It has recently become clear, however (Goldberg, Dodson and Muller, r954; Dodson, I955), that an important contributing factor to the diversity is the location of the flare on the solar disk. For example, flares near the limb show, on the average, much wider $\mathrm{H} \alpha$ near maximum than those near the centre of the disk, and the very widest lines observed by Ellison are found to occur only near the limb. It would seem that the centre-limb effects revealed by the $\mathrm{H} \alpha$ line can contribute important information on the physical nature of flares. Just as centre-to-limb observations in the Fraunhofer lines and continuum reveal the structure of the photosphere, so may similar observations perhaps give an inkling of the vertical temperature distribution in flares.

Unfortunately, the same flare cannot be observed both at the centre of the disk and at the limb. However, sufficient observational data exist to reveal certain statistical features of the centre-limb differences:

(I) As pointed out above, the $\mathrm{H} \alpha$ line width is on the average greater near the limb than at the centre of the disk.

(2) Many of the $\mathrm{H} \propto$ profiles observed by Ellison and also by Mustel and Severny exhibit strong central self-reversals. Miss Dodson has called my attention to the fact that the most pronounced self-reversals are always found near the limb.

(3) According to Miss Dodson, the average $\mathrm{H} \alpha$ central intensity, in absolute units, decreases with increasing central meridian distance according to the relationship

$$
I(\theta)=0 \cdot 63+0 \cdot 34 \cos \theta .
$$

(4) $\mathrm{H} \alpha$ light curves by Miss Dodson reveal that flares with the slowest rates of rise and decline are observed only in the central part of the solar disk and that flares with the fastest decline occur only near the limb.

The centre-limb difference between the rates of rise and decline of the light curves suggests that the upper portions of the flare layer behave differently from the lower regions. If the optical thickness in the centre of $\mathrm{H} \alpha$ for the high layers is small when the flare is viewed at the centre of the disk, most of the radiation will come from the lower portions. Near the limb the optical thickness may be increased by the sec $\theta$ factor to the point where the observed central $\mathrm{H} \alpha$ radiation originates almost entirely from the high layers. Further, the fact that the high layers are relatively cool is suggested both by the decrease in central intensity and by the exaggerated nature of the central self-reversal towards the limb.

In all probability there is not a sharp temperature boundary between the upper and lower flare layers, but rather a continuous gradient of temperature. For exploratory purposes, however, it will be sufficient to visualize the flare as consisting of two isothermal layers, the upper layer being cooler than the lower. Let the lower and upper layers be denoted by the subscripts $I$ and 2, respectively. It is assumed that the two layers are plane parallel in form, that their tangential extent is large compared with their thickness, and that they are located immediately above the photosphere. The radiation emerging from the layers in the neighbourhood of $\mathrm{H} \alpha$ is made up of three parts: (I) the residual radiation from the underlying $\mathrm{H} \alpha$ absorption profile, as modified by absorption in the flare layers; (2) the emission from layer I, as modified by absorption in layer 2 ; and (3) the emission from layer 2. The expression for the emergent intensity is therefore as follows:

$$
\begin{gathered}
E_{\nu}(\theta)=A_{\nu}(\theta) e^{-\left(\tau_{1}+\tau_{\imath}\right) \sec \theta}+P_{1}\left(\mathrm{I}-e^{-\tau_{1} \sec \theta}\right) e^{-\tau_{\mathbf{z}} \sec \theta}+P_{2}\left(\mathrm{I}-e^{-\tau_{\mathbf{1}} \sec \theta}\right) . \\
656
\end{gathered}
$$


In equation (I) $A_{\nu}$ is the residual intensity in the $\mathrm{H} \alpha$ absorption profile, which is a function of $\theta$, the central meridian distance, $P_{1}$ and $P_{2}$ are the Planck intensities corresponding to the Boltzmann temperatures of layers $I$ and 2 , respectively, while $\tau_{1}$ and $\tau_{2}$ are the optical depths at the centre of $\mathrm{H} \alpha$ for layers I and 2, respectively.

One of the most striking examples of a flare with a strong central self-reversal in $\mathrm{H} \alpha$ occurred on II February I949, and was observed by Ellison (I950). The position of the flare on the disk was $\mathrm{S} .10^{\circ}, \mathrm{W} .80^{\circ}$ and hence $\sec \theta=5.75$. The observed central intensity was about $I \cdot 0$, in units of the local continuum, but about I $\AA$ to the violet, the intensity increased to $I \cdot 8$. It was found that equation ( $I$ ) gave a good representation of this profile, with a maximum error of about I0 $\%$, when the following values of the parameters were employed: $P_{1}=\mathrm{I} \cdot 8, P_{2}=\mathrm{I} \cdot 0, \tau_{1}=4 \cdot \mathrm{I} \times \mathrm{IO}^{3}$, and $\tau_{2}=\mathrm{ro}$. It was further assumed that the line was broadened by combined Doppler effect and radiation damping, the Doppler width corresponding to a kinetic temperature of $8000^{\circ}$. Since the absorption coefficient per atom at the line centre is $5.5 \times 10^{-13}$, an optical depth of $4 . \mathrm{I} \times 1 \mathrm{O}^{3}$ is equivalent to $7.5 \times \mathrm{IO}^{15}$ two-quantum atoms per $\mathrm{cm}^{2}$ in the line of sight in the radial direction. The corresponding value found by Mustel and Severny for the great flare of 5 August I949 is $6.5 \times \mathrm{IO}^{15}$.

It was then considered of interest to calculate $\mathrm{H} \alpha$ profiles for the same flare observed at three different positions on the disk, namely, $\cos \theta=I \cdot 0,0.3$ and 0.14 , the values of $P_{1}$ and $P_{2}$ being held at $\mathrm{r} \cdot 8$ and $\mathrm{I} \cdot 0$, respectively, for $\cos \theta=0 \cdot \mathrm{I} 4$. Owing to limbdarkening in the continuum, the $\mathrm{H} \alpha$ intensity from a given flare, in units of the neighbouring continuum, will be half as great at $\cos \theta=\mathrm{I} \cdot 0$ as at $\cos \theta=0 \cdot \mathrm{I}_{4}$. The values of $P_{1}$ and $P_{2}$ for a given flare are therefore inversely proportional to the percentage limb darkening. The calculations were carried out with $\tau_{1}$, as before, equal to $4 \cdot \mathrm{I} \times \mathrm{IO}^{3}$, but with three different values of $\tau_{2}$, namely $O \cdot I, I \cdot O$, and Io. There is a striking dependence of the profiles upon $\theta$. This is due in part to the $\sec \theta$ factor in the optical depth, and in part to the limb-darkening, which greatly enhanced the apparent flare intensity at the limb.

It is of great interest to note that if the line-width were measured visually with the spectrohelioscope, the profiles at the centre disk would give a width of about $2 \AA$, but at $\cos \theta=0.14$ the width would be at least $8 \AA$. It is further to be noted that the central self-reversals are much enhanced at the limb, especially for the smaller values of $\tau_{2}$.

Finally, the observed limbward decrease of the absolute central intensity may also be explained by the two-layer hypothesis, provided that $\tau_{\mathbf{2}}$ is small but appreciable. To illustrate, the $\mathrm{H} \alpha$ profiles have been re-plotted with intensities relative to that of the continuum at the centre of the disk, and for $\tau_{2}=0 . I$ and $I \cdot 0$. When $\tau_{2}=0 . I$, the line centre is not saturated and hence decreases in intensity with decreasing $\cos \theta$, the reduction at $\cos \theta=0 \cdot \mathrm{I}_{4}$ being about $25 \%$. When $\tau_{2}=\mathrm{I} \cdot 0$, the line centre is saturated at $\cos \theta=0.3$ and no further decrease in central intensity occurs as $\cos \theta$ becomes smaller.

\section{DISCUSSION}

C. DE JAGER: What mechanism has been adopted for the widening of $\mathrm{H} \alpha$ in the flare?

L. GoLDBERG: It is assumed that the line is widened by Doppler broadening with a kinetic temperature of $8000^{\circ} \mathrm{K}$. and by radiation damping in the wings.

C. DE JAGER: Can the increase in $\mathrm{L} \alpha$ emission by a factor $\mathrm{rO}^{7}$ be explained?

L. GoldBERG: The L $\alpha$ intensity during flares has not yet been observed and estimates based upon ionospheric data are conflicting.

M. A. Ellison: I suppose your model refers to a flare emission region with horizontal extension and small vertical thickness. If the flare is mainly filamentary this may modify your conclusions to some extent. 
L. Goldberg: The model can easily be modified in this direction. Contrary to my earlier belief, the most important factor in the widening of the profile at the limb is the limb darkening in the continuum rather than the $\sec \theta$ factor.

H. ALFVEN: Which gas density has been assumed?

L. GOLDBERG: It is not necessary at this time to make assumptions as to the gas density, since only radiation damping is required to account for the wing broadening. The theory gives only the total number of two-quantum atoms in the line of sight and the values obtained agree closely with those found by Mustel and Severny.

\section{FLARE-ASSOCIATED SOLAR PHENOMENA}

\section{By W. O. RoBERTS}

Flares are the most abrupt, and in many ways the most spectacular, of solar phenomena. Thus, it is not surprising that associated with flares are a number of other optically observed effects in the solar corona, prominences and plages.

As Dr Ellison states and Dr McMath's superb films show, flares almost always arise from plages(x) and they are most likely to occur in unusually bright or fluctuating plages associated with complex or bipolar sunspot groups, particularly in the first few days of development of spot groups when the plages are usually luminous. Sometimes the occurrence of a flare leaves the plage brighter and larger than it was before the flare (2). Strangely enough, flares rarely show any associated photospheric effects. A few years ago some workers felt that there was an area reduction in sunspots immediately after the outbreak of flares, but Newton and Howe have now shown that there appears to be no such area discontinuity, either in umbra or penumbra, at the time of flares (3).

Flares are generally accompanied by high-speed surge prominences that are sometimes of spectacular behaviour (4). They are visible in hydrogen as 'high-speed dark filaments' on the solar disk, or at the limb as surge prominences. Many of these rise to heights as great as 100,000 km. and some achieve velocities of over $500 \mathrm{~km}$./s. Typical flare surges appear at the time of maximum intensity of the flare, and are already rapidly moving when first observed. Often they decelerate under the influence of forces that exceed solar gravity, finally coming to rest, and then returning to the photcsphere along the trajectory they had followed in their outwards course. It is widely speculated that surge prominences are clouds of hydrogen gas set into motion by absorption of the powerful blast of $\mathrm{L} \alpha$ radiation that results from the main 'flash' of the flare maximum. The initial point at which such surges appear is generally near the flare, but not directly over it.

Dr Bruzek has done so much important work on the effects of flares on other solar phenomena that I feel it should be he rather than I who is giving this paper. He has, for example, shown(s) from the observations made at Kanzelhöhe that dark hydrogen filaments, seen in projection on the solar disk, often feel the effects of solar flares, even when they are at distances as great as several hundred thousand kilometres from the flares. Such filaments can be 'activated' by some invisible disturbance that appears to propagate from the flare with velocities from about 20 to about $200 \mathrm{~km}$. $/ \mathrm{s}$. When activated, the filaments become darker and larger, display sudden changes of shape and position and develop line-of-sight velocities. Sometimes, in cases of nearby dark filaments, a travelling disturbance can be detected propagating along the length of the filament. In other cases, the activated filament may lie at a substantial distance, and may possess no obvious association with the spot group in which the flare has occurred, so that its activation takes place some time later, and might, therefore, easily be thought an independent event. In some cases the flare-activation of a dark filament may lead to its destruction. On the other hand, nearby dark filaments sometimes exhibit little or no response to flares. 